CLINICAL ETHICS

\title{
Evaluation of end of life care in cancer patients at a teaching hospital in Japan
}

\author{
Y Tokuda, N Nakazato, K Tamaki
}

J Med Ethics 2004;30:264-267. doi: 10.1136/jme.2003.000125

See end of article for authors' affiliations

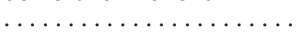

Correspondence to: Yasuharu Tokuda, MD,

Division of General Internal Medicine, Department of Medicine, Okinawa Chubu Hospital, Gushikawa City, Okinawa 904-2293, Japan; tokuda_yasuharu@ hosp.pref.okinawa.jp

Revised version received 9 April 2002

Accepted for publication

26 January 2003

\begin{abstract}
Objectives: To analyse the decision making for end of life care for patients with cancer at a teaching hospital in Japan at two periods 10 years apart.

Design and setting: Retrospective study conducted in a 550 bed community teaching hospital in Okinawa, Japan.

Patients: There were 124 terminally ill cancer patients (45 women; 79 men; median age, 69 years) admitted either in 1989 and 1999 for end of life care with sufficient data to permit analysis.

Main measurements: Basic demographic data, notification to the patient that he or she had cancer, patient involvement in do not resuscitate (DNR) orders, and various medical interventions which were performed in the month prior to the patient's death were evaluated.

Results: In 1989 none of the patients were notified of their diagnosis; in 1999 five patients were informed $(p=0.026)$. Of the $113(91 \%)$ patients with a written DNR order, none were involved in consenting to the DNR order. In the month before death, patients in both groups received non-palliative treatments such as feeding tube placements (five in 1989; five in 1999), total parenteral nutrition (six in 1989; eight in 1999), and intravenous albumin infusion (four in 1989; five in 1999). Morphine use increased (30\%) significantly in 1999 compared with the 1989 group.

Conclusions: The majority of patients dying of cancer were still not informed of their diagnosis and were seldom involved in DNR decision making at a teaching hospital in Japan. There was no change in the number of potentially futile interventions that were performed (6-13\%) but morphine use increased. Modern ethical education is urgently needed in Japanese medical practice to improve decision making process in the end of life care.
\end{abstract}

$\mathrm{P}$ alliative care is the most appropriate management strategy for patients with incurable cancer in terminal stage. ${ }^{12}$ However, most Japanese cancer patients seemed to die in a hospital without being informed of their diagnosis. Non-palliative and futile treatments seem to be performed frequently during patients' last days. ${ }^{3}$ Although many Japanese physicians write do not resuscitate (DNR) orders for patients with terminal diseases,${ }^{4}$ there has been comparatively little discussion of DNR policy and standardisation. There also seems to be inadequacies in the use of DNR orders because patients are not involved in the DNR decision making. ${ }^{45}$

The past decade has seen several ethical proposals to improve end of life care from various physician organisations and patient societies in Japan. ${ }^{6-9}$ They emphasise the need to obtain informed consent, ${ }^{6}{ }^{10}$ and to develop the hospice network to provide sophisticated palliative care ${ }^{89}$ for patients with terminal cancer in Japan. Therefore, to better describe the types of care such patients received in a general hospital in Japan over the last decade, a study was conducted by a retrospective chart review of the end of life decision making and the non-palliative treatments given to two groups of patients with terminal cancer in 1989 and in 1999.

\section{MATERIALS AND METHODS}

This is a retrospective descriptive study. In January to December 1989, and January to December 1999, 124 consecutive deaths of patients with terminal cancer admitted to our 550 bed community based teaching hospital were studied. This hospital is located in rural part of Okinawa, a remote tropical island in southern Japan. All patients had detailed medical records, including the sites of cancers, the circumstances surrounding their notification of having terminal cancer, and details of end of life care.

Cognitive competency in individual patients was assessed by the attending resident or staff physician and recorded in medical charts. If patients had psychosis, delirium, dementia, or depression over half of the hospitalised period, their cognitive function was judged to be not mentally competent for decision making for end of life care.

Informed consent for participation in this study could not be obtained from all patients since most of them were not informed about having terminal cancer. However, family or next of kin of all patients had patient's diagnosis, treatment plan, expected therapeutic efficacy, and projected survival formally explained in detail, culminating in them allowing surrogate consent for this retrospective study.

Ethics approval for this study was given by the Okinawa Chubu Hospital Institutional Review Board.

Cardiopulmonary resuscitation (CPR) is defined as application of external chest compression and rescue breathing. The DNR document is not considered a legal document in Japan, but providing such a document in a medical chart implies a prohibition of external chest compression, intratracheal intubation, and artificial ventilation.

Several medical interventions with futile potentiality were selected in considering their possible effectiveness in palliative care. They included total parenteral nutrition, intravenous albumin infusion, and tube feeding during the last four weeks before death. ${ }^{11}$ Their medical indication and actual use was totally dependent on the attending resident or staff physician in individual patients. It was not usual practice in this hospital for patients and the family to specifically request these treatment measures. None of the investigators were involved in this decision process. 
Statistical methods were used to analyse the difference of variables that were important in evaluation about truth telling for patient having terminal cancer, DNR status and its decision making process, and medical interventions. Fisher's exact test was performed and it was considered significant if $\mathrm{p}<0.05$.

\section{RESULTS}

Baseline characteristics of patients are listed in table 1. Patients ranged in age from 20 to 97 years. Most were married with children. Educational level ranged from illiteracy to college and beyond, but well over $80 \%$ of patients had received formal education. Religious affiliation for almost all the patients was the Okinawan traditional version of Buddhism. Japanese was the spoken tongue for all patients, although some elderly patients identified Okinawan dialect as their first language. Financial status was difficult to assess, but treatment was never withdrawn for economic reasons. There is universal health insurance coverage for all citizens of Japan.

All patients were in the terminal stage of their diseases, with a poor performance status. Patient diagnoses included 44 gastrointestinal cancers, 27 lung cancers, 19 haematological cancers, and 5 breast cancers, and other varieties of cancers (table 1). The immediate cause of death could be attributed directly to progressive cancer in $95 \%$ of patients, with seven patients dying from sepsis.

At the point when performance status became poor around ECOG (Eastern Cooperative Oncology Group) class 4 for a particular patient, ${ }^{12}$ the family members, but seldom the patient directly, would be informed of the seriousness of the situation and encouraged to make future arrangements. There was a family meeting session usually lasting 30$40 \mathrm{~min}$. In this session, DNR status was discussed with the rationale that $\mathrm{CPR}$ would be futile as a result of progressive cancer.

Table 2 compares characteristics of decision making for end of life care in 1989 and 1999. Rate of cognitive competency in patients was not different between 1989 and 1999. While none of the patients in 1989 were notified of having terminal cancer, five patients $(8.2 \%)$ in 1999 were informed of their diagnosis $(p=0.026)$. Most patients who were not told of their diagnosis had been given provisional explanation, such as chronic progressive illness and benign but inoperable mass lesions. The age range of patients who were informed in 1999 was 44-49 years old. This means that relatively young patients tended to be informed of their diagnosis.

None of the patients signed consent orders to forgo CPR. DNR orders were signed prior to death by attending residents or staff physicians with median of 10 days. Although discussions with family about DNR were recorded in the medical charts of almost all patients, there were no formal DNR consent documents for the families to sign, and so there

Table 1 Baseline characteristics of patients

\begin{tabular}{llll}
\hline Year & $\begin{array}{l}1989 \\
\mathbf{n}=63(\%)\end{array}$ & $\begin{array}{l}1999 \\
\mathbf{n}=61(\%)\end{array}$ & $\mathbf{p}$ Value \\
\hline Female & $20(32)$ & $25(41)$ & 0.351 \\
Median age (range) & $69(20-97)$ & $69(39-95)$ & 0.41 \\
Sites of cancer & & & 0.625 \\
$\quad$ Lung & $13(20.6)$ & $14(23.0)$ & \\
$\quad$ Colorectal & $11(17.5)$ & $5(8.2)$ & \\
Oesophagus & $7(11.1)$ & $3(4.9)$ & \\
ATL & $6(9.5)$ & $7(11.5)$ & \\
Stomach & $4(6.3)$ & $4(6.6)$ & \\
Others & $22(35)$ & $28(46)$ & \\
\hline ATL, adult T cell leukaemia. & & \\
\hline
\end{tabular}

Table 2 Characteristics of end of life decision making

\begin{tabular}{llll}
\hline Characteristics & $\begin{array}{l}1989 \\
\mathbf{n}=63(\%)\end{array}$ & $\begin{array}{l}1999 \\
\mathbf{n}=61(\%)\end{array}$ & $\mathbf{p}$ Value \\
\hline Cognitive competency & $48(76.2)$ & $49(80.3)$ & 0.665 \\
Yes & $15(23.8)$ & $12(19.7)$ & \\
$\quad$ No & 0.026 \\
Informed of having cancer & $0(0)$ & $5(8.2)$ & \\
Yes & $63(100)$ & $56(91.8)$ & \\
No & $56(88.9)$ & $57(93.4)$ & 0.53 \\
DNR order & $7(11.1)$ & $4(6.6)$ & \\
$\quad$ Yes & $9(14.3)$ & $6(9.8)$ & \\
No & $54(85.7)$ & $55(90.2)$ & \\
CPR performed & & \\
Yes & No &
\end{tabular}

are no consent records signed by the families. None of the patients had made a living will or advance directive.

In two patients (one in 1989 and one in 1999) where the attending resident had not signed DNR status, resuscitation was not performed because it was judged to be futile by the on call medical staff. CPR was instituted in 15 patients, mostly at the insistence of the family. Six patients (three in 1989 and three in 1999) received CPR by request of family despite the presence of a DNR order. To avoid any potential future litigation by the family, CPR was performed for at least $30 \mathrm{~min}$ in this hospital. None of the patients lived to hospital discharge.

Almost all patients experienced moderate or severe pain. Morphine was given significantly more often in patients in 1999; $38(62 \%)$ of the patients in 1999 received morphine compared with $19(30 \%)$ of patients in $1989(\mathrm{p}=0.001)$. All patients had intravenous access for provision of fluids if nutrition by mouth or via nasogastric tube was not possible. There was no significant difference between the two groups for administration of antibiotics (26 in 1989 and 32 in 1999, $\mathrm{p}=0.28$ ) and blood transfusion (11 in 1989 and 12 in 1999, $\mathrm{p}=0.82$ ).

Potentially futile medical interventions were occasionally employed at the last four weeks to death. Specific treatments are given in table 3. Analysis using the Fisher's exact test shows that there is no difference 10 years apart in nonpalliative medical interventions including total parenteral nutrition, intravenous albumin infusion, and tube feeding.

\section{DISCUSSION}

In this retrospective, descriptive study, we found that only five patients $(4 \%)$ who died of cancer were informed of their diagnosis, although there was significant increase in the rate of them being informed of their diagnosis in 1999. DNR orders, which were documented in about $90 \%$ of cases, were written after getting consent, not by patients themselves, but by family or surrogates. CPR was performed in $12 \%$ ( 15 patients) of cases in this study. Moreover, our study demonstrates that patients with terminal cancer often received potentially futile non-palliative treatments during their last month of life.

Table 3 Number of patients receiving non-palliative treatments

\begin{tabular}{llll}
\hline Treatment & $\begin{array}{l}1989 \\
\mathbf{n}=63(\%)\end{array}$ & $\begin{array}{l}1999 \\
\mathbf{n}=61(\%)\end{array}$ & $\mathbf{p ~ V a l u e ~}$ \\
\hline $\begin{array}{l}\text { Total parenteral nutrition } \\
\text { Albumin infusion }\end{array}$ & $6(10)$ & $8(13)$ & 0.58 \\
Tube feeding & $4(6)$ & $5(8)$ & 0.741 \\
\hline
\end{tabular}


The disclosure of information to cancer patients is one of the most important issues in medical ethics. In Japan, few cancer patients were ever given a truthful statement of their diagnosis. Recently more and more patients are being informed of their diagnosis, as shown in our study. One survey reported a high rate $(71 \%$ in 1998) of information disclosure, ${ }^{13}$ while $13 \%$ of physicians informed patients with cancer of their disease in another survey. ${ }^{14}$ The disclosure rate of $8.2 \%$ in 1999 in our survey is still low in comparison to those reports. The reason for this low rate could be selection of the study location, Okinawa, which consists of rural islands far from mainland Japan and may be more traditional.

No patient personally signed his or her own DNR consent. Obviously, it is difficult for patients to participate in a decision about DNR orders when they do not know their diagnosis. In Japan, there was almost no consensus about whether to inform a patient of a diagnosis of cancer, and even the principle of informed consent may still be controversial. ${ }^{15-17}$ The Japanese patient may often be overprotected and precluded from knowing the reality of his or her terminal disease. There is also the family's fear for adverse emotional impact on patient's health if the complete truth were to be revealed. ${ }^{18}$ However, the previous study revealed that more than $60 \%$ of the public in Japan would prefer to be told of such a diagnosis. ${ }^{19}$ We believe that many patients wish to be so informed and to participate in decision making for their end of life care. There is growing effort for introducing the principle of informed consent in medical community. ${ }^{20} 21$

There is a rationale behind instituting a DNR order in patients with terminal cancer. ${ }^{22}$ Firstly, the patient will receive no medical benefit. Analysis has shown that patients with cancer who have poor functional status experience complete CPR failure with almost 100\% in-hospital mortality. Secondly, poor quality of life is expected after CPR. It suggests that CPR may also be avoided if there is poor quality of life before cardiopulmonary arrest. Lastly, a DNR order has the advantage of decreased mechanical ventilator support, decreased invasive and painful intervention for the patient, and in general, decreased economic burden. ${ }^{23}$

In general, patients in Japanese hospitals participated in the decision about their own DNR orders for themselves at a lower rate (5\%) than patients in other countries (14-41\%). ${ }^{24-28}$ Patients in Japan have traditionally tended to entrust their most important treatment decisions to physicians. However, the absence of patient's DNR consent raises the question of whether the attending resident or staff physician decisions fundamentally reflect wishes and opinions of the patient. Documentation of such consent may play only a superficial role in averting unnecessary CPR.

An important factor in omission of DNR discussion with patient may lie also with the medical staff. ${ }^{29}$ Physicians usually discuss end of life decisions with surrogates rather than with the patients themselves. They are often reluctant to have discussions of CPR and DNR, fearing potential adverse effects on patients, and that patients may lose hope in the doctors' capacity to heal them. They perceive such discussions as filled with foreboding of the worst. Obtaining a DNR status may take more than $30 \mathrm{~min}$ and the discussion often has to be repeated, which poses a burden upon busy hospital practice. ${ }^{30}$ Staff physicians and residents could institute DNR order without consulting the family when the physician feels that a CPR is unjustified and futile, if the hospital and the patient care committees have policies and procedures to back such decisions.

However, surveys showed that patients are willing and able to take part in such discussions in USA and even in Japan. ${ }^{31}$ Moreover, the Japan Society for Dying with Dignity published advice for physicians caring for terminally ill patients in 1992, suggesting that physicians take into account the wishes of patients, when the patient is competent. ${ }^{10}$ Good communication and discussion is essential between patient, family, religious representative, and hospital staff to clarify the patient's preferences if the patient is still mentally competent. ${ }^{2}$ Moreover, the Yokohama District Court found that life sustaining treatment can be ended if either a terminally ill patient or the patient's family, acting on their understanding of the patient's wishes, requests it. ${ }^{32}$

There has been a lack of explicit, published guidelines for DNR orders in Japan. However, a structured DNR order sheet may help in clarifying the finer details of the DNR order for the patient, patient surrogate, and hospital staff. ${ }^{5}$ Formal physician education is urgently needed to improve the frequency, quality, and timing of these discussions in curriculum of undergraduate and postgraduate medical education. Physicians should educate terminally ill patients about their wishes with regard to life sustaining treatment and provide psychosocial support so that the patients feel comfortable expressing their preferences. ${ }^{33}$

Many cancer patients who die in a teaching hospital in Japan receive non-palliative treatments, most of which have the potential to be futile. It is difficult to determine the extent to which the quality of life of the patients was more respectfully considered in recent years since medical interventions with potential futility were still employed without change in the trend between the two study periods.

Our study shows that $11 \%$ of the patients received total parenteral nutrition during their terminal hospitalisation and $8 \%$ had feeding tube placement. Nutrition and hydration through artificial means (intravenous fluids, nasogastric, gastrostomy, or jejunostomy tubes; total parenteral nutrition) can be administered to terminally ill patient. However, when a patient has greatly reduced fluid intake related to the normal dying process, fluids can be given in small amounts by mouth as tolerated to prevent dryness of throat. Invasive procedures such as intravenous therapy may be considered when such treatment may enhance the comfort of the patient more than non-invasive alternatives. ${ }^{2}$

In our study, there were $7 \%$ of patients who received intravenous albumin infusion. Recent systematic review of randomised controlled trials found no evidence that albumin reduced mortality and a strong suggestion that it might increase the risk of death in patients with hypovolaemia, burns, or hypoproteinaemia. Overall, the risk of death in patients treated with albumin was 6\% (95\% confidence interval 3 to 9) higher than in patients not given albumin. ${ }^{34}$ Therefore, even in the presence of hypoalbuminaemia, the use of human albumin in the management of terminally ill patients should be discouraged.

Our data should be interpreted in light of several limitations inherent in the study design. Firstly, our study was performed in a single hospital, and the results may not be generalisable to other hospitals. Secondly, the study was retrospective in design so that prospective study by validation cohort seems mandatory.

With an increasing number of hospices being established locally, and an increasing popularity of the concept of dignified death in Japan, it is hoped that the place of dying will shift from major hospitals to hospice units. Both patient and medical staff should be more aware of end of life decision making and more accepting of earlier DNR discussions involving the patient personally. Also, it is necessary to establish evidentiary standard for the withholding of life prolonging treatments for terminally ill patients regarding total parental nutrition (TPN), albumin infusion, and tube feeding. Adopting a patient centred method such as informed 
consent to DNR order and avoiding futile interventions would be an important step to improve the end of life care in Japan. ${ }^{35}$

\section{ACKNOWLEDGEMENTS}

We thank Dr Suresh K Joishy, MD, for in depth review of the manuscript.

\section{Authors' affiliations}

Y Tokuda, N Nakazato, K Tamaki, Division of General Internal Medicine, Department of Medicine, Okinawa Chubu Hospital,

Okinawa, Japan

Funding: none.

Conflict of interest: none.

\section{REFERENCES}

1 Joishy SK. Palliative medical oncology. Surg Oncol Clin N Am 2001; 10:203-20.

2 Joishy SK. Palliative medicine secrets. Philadelphia: Hanley \& Belfus, 1999:5-20.

3 Kai I, Ohi G, Yano E, et al. Communication between patients and physicians about terminal care: a survey in Japan. Soc Sci Med 1993;36:1151-9.

4 Arai T, Namiki A, Amaha K, et al. Response to a questionnaire on DNR-order from 307 trustee members of Japanese medical societies. Masui 1994:43:600-11.

5 Fukaura A, Tazawa H, Nakajima H, et al. Do-not-resuscitate orders at a teaching hospital in Japan. N Engl J Med 1995;333:805-8.

6 The Bioethical Committee for Japanese Medical Association. Special report about "what physicians faced with patients with terminal illness should do" [Japanese]. Nippon Ishikai Zassi 1992;107:1209-17.

7 The Japan Society for Dying with Dignity. Death with dignity [Japanese] Tokyo: Houken, 1998:391.

8 Hospice care research institute. Design of hospice, 2nd ed [Japanese]. Tokyo: Miwashoten, 1993.

9 The Japan Hospice/Palliative Care Foundation. www.hospat.org.

10 The Japan Society for Dying with Dignity. Report for physicians [Japanese] Tokyo, 1992

11 Ahronheim JC, Morrison RS, Baskin SA, et al. Treatment of the dying in the acute care hospital. Advanced dementia and metastatic cancer. Arch Intern Med 1996;156:2094-100.

12 Roila $F$, Lupattelli $M$, Sassi $M$, et al. Intra and interobserver variability in cancer patients' performance status assessed according to Karnofsky and ECOG scales. Ann Oncol 1991:2:437-9.

13 Horikawa N, Yamazaki T, Sagawa M, et al. Changes in disclosure of information to cancer patients in a general hospital in Japan. Gen Hosp Psychiatry 2000;22:37-42.
14 Tanida N. Japanese attitudes towards truth disclosure in cancer. Scand J Soc Med 1994;22:50-7.

15 Murakami K, Haga T. The protocol of informing cancer patients of their condition. Gan To Kagaku Ryoho 1986;13:2693-8.

16 Teshima $\mathrm{H}$, Kihara $\mathrm{H}, \mathrm{Ago} \mathrm{Y}$, et al. Terminal care in patients with lung cancer. Nihon Kyobu Shikkan Gakkai Zasshi 1987;25:305-11.

17 Murakami K, Haga T. Cancer treatment in terminal stage. Gan To Kagaku Ryoho 1989:16:740-5.

18 Akabayashi A, Fetters MD, Elwyn TS. Family consent, communication, and advance directives for cancer disclosure: a Japanese case and discussion. J Med Ethics 1999;25:296-301.

19 The Prime Minister's Office, Division of Public Information. The publicopinion poll, vol 23, no 5 [Japanese]. Tokyo: Ministry of Finance Printing Office, 1991:46-57.

20 Leflar RB. The cautious acceptance of informed consent in Japan. Med Law 1997:16:705-20.

21 Akabayashi A, Fetters MD. Paying for informed consent. J Med Ethics 2000;26:212-14

22 Tomlinson $\mathrm{T}$, Brody $\mathrm{H}$. Ethics and communication in do-not-resuscitate orders. N Engl J Med 1988;318:43-6.

23 Wenger NS, Pearson ML, Desmond KA, et al. Outcomes of patients with donot-resuscitate orders. Toward an understanding of what do-not-resuscitate orders mean and how they affect patients. Arch Intern Med 1995; 155:2063-8.

24 Jonsson PV, McNamee M, Campion EW. The 'do not resuscitate' order. A profile of its changing use. Arch Intern Med 1988;148:2373-5

25 Schwartz DA, Reilly P. The choice not to be resuscitated. J Am Geriatr Soc 1986:34:807-11.

26 Gleeson K, Wise S. The do-not-resuscitate order. Still too little too late. Arch Intern Med 1990;150:1057-60.

27 Berlowitz DR, Wilking SV, Moskowitz MA. Do-not-resuscitate orders at a chronic care hospital. J Am Geriatr Soc 1991;39:472-6.

28 Bedell SE, Pelle D, Maher PL, et al. Do-not-resuscitate orders for critically ill patients in the hospital. How are they used and what is their impact? JAMA 1986;256:233-7.

29 Lofmark R, Nilstun T. Do-not-resuscitate orders - should the patient be informed? J Intern Med 1997;241:421-5.

30 Smith TJ, Desch CE, Hackney MH, et al. How long does it take to get a "do not resuscitate" order? J Palliat Care 1997;13:5-8.

31 Heffner JE, Barbieri C, Casey K. Procedure-specific do-not-resuscitate orders. Effect on communication of treatment limitations. Arch Intern Med 1996; 156:793-7

32 Anon. Summary of a judgment in a case of an euthanasia at Tokai University Hospital [Japanese]. Asahi Shimbun 1995;28:3

33 Stolman CJ, Gregory JJ, Dunn D, et al. Evaluation of the do not resuscitate orders at a community hospital. Arch Intern Med 1989;149:1851-6.

34 Cochrane Injuries Group Albumin Reviewers. Human albumin administration in critically ill patients: systematic review of randomised controlled trials. BMJ 1998;317:235-40.

35 Tokuda Y. Teaching ethics in Japan. Lancet 1995;345:1574. 